Ger J Exerc Sport Res 2018 • 48:467-477 https://doi.org/10.1007/s12662-018-0535-y Received: 23 March 2018

Accepted: 25 July 2018

Published online: 15 August 2018

(c) The Author(s) 2018

CrossMark

\author{
Anika Frühauf ${ }^{1}$ (D) Will A. S. Hardy ${ }^{2} \cdot$ Ross Roberts $^{2} \cdot$ Martin Niedermeier' $^{1}$ \\ Martin Kopp ${ }^{1}$ \\ ' Department of Sport Science, University of Innsbruck, Innsbruck, Austria \\ ${ }^{2}$ Institute for the Psychology of Elite Performance, School of Sport, Health, and Exercise Sciences, Bangor \\ University, Bangor, UK
}

\title{
Structural validation of three German versions of behavioral and motivational scales in high- risk sports
}

Suggesting that sensation seeking is the single panoptic explanation for voluntary engagement in sports as diverse as Himalayan mountaineering (e.g., extended duration, long periods of boredom, physically painful) and skydiving (e.g., very limited duration, characterized by thrilling enjoyable sensations) seems overly simplistic and several studies have shown that the motives for participation in high-risk sport are more varied than this (Barlow et al., 2015; Barlow, Woodman, \& Hardy, 2013; Castanier, Le Scanff, \& Woodman, 2010; Castanier, Le Scanff, \& Woodman, 2011; Frühauf, Hardy, Pfoestl, Hoellen, \& Kopp, 2017; Kerr \& Houge Mackenzie, 2012; Lafollie \& Le Scanff, 2007; Woodman et al., 2013; Woodman, Hardy, Barlow, \& Le Scanff, 2010; Woodman, Huggins, Le Scanff, \& Cazenave, 2009). A number of qualitative studies have uncovered additional and alternative motives for participation in high-risk sports (e.g., emotion regulation, agency, challenge, nature) (Brymer, 2010; Brymer \& Gray, 2010; Frühauf et al., 2017; Kerr \& Houge Mackenzie, 2012; Willig, 2008; Woodman et al., 2010). In light of these developments in understanding the motivations for participation in high-risk sports, a number of quantitative tools have been developed.

Barlow et al. (2013) established the Sensation Seeking, Emotion Regulation and Agency Scale (SEAS), a series of inventories that measure the following: the need for sensation, difficulty with emotion regulation, and lack of agency between bouts of participation in high-risk sports; the experience of sensation, emotion regulation, and agency while participating; and the transfer of sensation, emotion regulation, and agency following participation. This was based on research showing that participants in prolonged high-risk activities have difficulty with emotion regulation and a diminished sense of agency in aspects of their life and thus might participate in those high-risk sport activities to experience agency and become aware of their emotions (Woodman et al., 2010). Barlow et al. (2013) developed the SEAS using a variety of participants who took part in both high-risk sports (e.g., mountaineering and skydiving) and low-risk sports (e.g., basketball and hockey), in doing so they found that some activities might be motivated by the sensations of the activity (e.g., skydiving) and others might be motivated by the emotion regulation and agency transfers (e.g., mountaineering).

Understanding the motives for participation in high-risk sports allows researchers to better comprehend the potential benefits and risks to participants. Nevertheless, the objective risk of the activity is undeniable, which is underlined by the higher rates of both acci- 
Table 1 Bayesian structural equation modelling (BSEM) fit and convergence

\begin{tabular}{|c|c|c|c|c|c|c|c|}
\hline \multirow[b]{2}{*}{ Scale } & \multirow[b]{2}{*}{$\begin{array}{l}\text { No. of free } \\
\text { parameters }\end{array}$} & \multirow[b]{2}{*}{ DIC } & \multirow[b]{2}{*}{ PPp } & \multicolumn{2}{|c|}{$\begin{array}{l}\text { Difference between } \\
\text { observed and repli- } \\
\text { cated } \chi^{2} 95 \% \text { credi- } \\
\text { bility interval }\end{array}$} & \multirow[b]{2}{*}{$\begin{array}{l}\text { Itera- } \\
\text { tions } \\
\text { PSR > 1.1 }\end{array}$} & \multirow[b]{2}{*}{$\begin{array}{l}\text { Final } \\
\text { PSR }\end{array}$} \\
\hline & & & & $\begin{array}{l}\text { Lower } \\
2.5 \%\end{array}$ & $\begin{array}{l}\text { Upper } \\
2.5 \%\end{array}$ & & \\
\hline $\begin{array}{l}\text { G-SEAS Be- } \\
\text { tween }\end{array}$ & 164 & $20,934.814$ & 0.506 & -44.003 & 42.693 & 45,800 & 1.016 \\
\hline G-SEAS While & 164 & $24,762.467$ & 0.507 & -43.554 & 42.348 & 74,100 & 1.039 \\
\hline $\begin{array}{l}\text { G-SEAS After } \\
\text { 3-factor }\end{array}$ & 164 & $19,096.132$ & 0.512 & -44.497 & 42.766 & 33,500 & 1.012 \\
\hline $\begin{array}{l}\text { G-SEAS After } \\
\text { 2-factor }\end{array}$ & 148 & $19,097.742$ & 0.517 & -44.149 & 42.083 & 44,700 & 1.039 \\
\hline G-ACCSI & 40 & $10,168.852$ & 0.490 & -20.447 & 21.182 & 67,800 & 1.009 \\
\hline G-RTI & 50 & $11,324.093$ & 0.514 & -23.794 & 23.269 & 68,200 & 1.028 \\
\hline
\end{tabular}

dents and close calls ${ }^{1}$ in high-risk sports than in low-risk sports (Barlow et al., 2015; Gosteli et al., 2016). To contribute towards more safety in high-risk sports two further aspects have to be taken into account, namely objective risks and participants' behavior.

Objective risks (e.g., environmental hazards such as avalanches) must be accepted as an inherent aspect of participation in high-risk sport, but participants are not risk-takers per se since they are able to influence their risk exposure by adapting their behavior (Gosteli et al., 2016; Leiter \& Rheinberger, 2016; Llewellyn \& Sanchez, 2008). Whereas objective risks cannot be modified, there seems to be an orthogonal nature of behavior in participants which consists of deliberate risk-taking and precautionary behavior (Woodman et al., 2013). To contribute to the understanding of these behaviors, the Risk-Taking Inventory (RTI) was developed to measure precautionary behavior (PB) and deliberate risk-taking (DRT) in high-risk sport participants (Woodman et al., 2013). Recent research suggests that behavior in highrisk sport, namely in freeriding, changes based on individuals' experiences of accidents and close calls (Frühauf et al.,

1 Close calls are defined as "incidents that come very close to resulting in a negative outcome" (Woodman etal., 2013, p.480)
2017). Thus, it is important to quantify accidents and close calls and relate them to participants' behaviors. This can be done by using the Accidents and Close Calls in Sports Inventory (ACCSI; Barlow et al., 2015). Research showed that accidents and close calls were positively correlated to DRT and negatively to PB (Barlow et al., 2015).

At present, SEAS, RTI, and ACCSI are available only in the English language and the scales were validated with English-speaking participants. However, there are differences in the amount of leisure time physical activity across European countries (Martínez-González etal., 2001) and there is also evidence for crosscultural differences in risk-taking (Mata, Josef, \& Hertwig, 2016) regarding risk behaviors like gambling or speed driving (Molinaro et al., 2014; Wallén Warner, Ozkan, \& Lajunen, 2009).

Furthermore, there is a dearth of validated measures for carrying out research in non-English-speaking high-risk sport populations. When considering that "The Alps comprise the largest and most popular sports region in Europe" (p. 1) and that many alpine sports are classified as high-risk sports (e.g., ski touring, mountaineering, mountain biking, rock and ice climbing and paragliding; Burtscher, 2008), it becomes clear that there is a need for validated measure for conducting research in non-English- speaking high-risk sport populations. Austria is just one German-speaking alpine country with almost one third of the $180,000 \mathrm{~km}^{2}$ of mountainous area in the Alps (Burtscher, 2008). Thus, with the aim of taking the first step towards filling the lacuna highlighted above the aim of the present research was to validate German language versions of the SEAS, RTI, and ACCSI.

\section{Method}

\section{Procedure}

Following institutional approval by the Board for Ethical Questions in accordance with the Declaration of Helsinki, we collected the data using a web-based questionnaire in a cross-sectional design. We recruited participants from a number of different high-risk sports via emails to students and employees of the University of the first author and local sports clubs (e.g., paragliding association). All participants completed the survey online. Participants not finishing the survey were excluded from analyses.

\section{Participants}

The final number of participants was 719 ( $25 \%$ female), with the highest numbers of individuals performing various disciplines in paragliding (59\%). Further, the sample consisted of freeride skiers (14\%), mountain trail runners ${ }^{2}(16 \%)$, freestyle skiers/snowboarders (7\%), as well as mountaineering athletes (4\%). The participants had a mean age of $35.4( \pm 11.6)$ years and reported an average of 7.3 $( \pm 6.1)$ years of experience. Age and experience varied between sport activities, with the youngest age and lowest years of experience in freestyle skiing and snowboarding (age: 23.0 \pm 3.8 years; years of experience: $6.0 \pm 3.6$ years) and the oldest age and most years of experience in paragliding (age: 38.9 \pm 11.4 years;

\footnotetext{
2 Mountain trail running as performed in the Alps is considered a high-risk sport since there is a risk of death and life-threatening accidents due to the exposure and steepness of the trails and/or sudden weather changes which occur in the mountains.
} 
years of experience: $7.9 \pm 6.5$ years). Female participation ranged from $19 \%$ in freestyle skiing/snowboarding to $32 \%$ in mountain trail running.

\section{Scales}

\section{Sensation Seeking, Emotion Regulation and Agency Scale (SEAS)}

The SEAS (Barlow et al., 2013) comprises three separate inventories which measure three different factors asking about three different times namely Between participation, While participating, and After participation. The Between participation inventory evaluates the time when not participating for a significant amount of time and measures need for sensation, difficulty with emotion regulation and lack of agency. The While inventory evaluates the experience of sensation seeking, emotion regulation and agency asking about the time while participating. The After inventory measures the transfer of sensation, emotion regulation, and agency asking about the time following participation. Each inventory contains 18 items with a seven-point Likert scale response mode ranging from one (completely disagree) to seven (completely agree).

Barlow et al. (2013) found evidence to support a three-factor structure for the Between and While inventories; however, they found that a two-factor model was a better fit to the data for the After inventory, with Agency and Emotion Regulation being combined into a single factor (i.e., agentic emotion regulation). Cronbach's alpha $(\alpha)$ displayed good internal consistency for each factor: Between participation inventory $(\alpha \geq 0.84)$, While participating inventory $(\alpha \geq 0.70)$, and After participation inventory $(\alpha \geq 0.89)$. The SEAS factors correlated with established measures of sensation seeking, emotion regulation and agency (Barlow et al., 2013).

\section{Risk-Taking Inventory (RTI)}

The RTI (Woodman et al., 2013) measures risk-taking in high-risk sports across two orthogonal factors, deliberate risk-taking (DRT, three items) and precautionary behaviors ( $\mathrm{PB}$, four items) on a seven item five-point Likert-scale

Ger J Exerc Sport Res 2018 · 48:467-477 https://doi.org/10.1007/s12662-018-0535-y

(C) The Author(s) 2018

A. Frühauf · W. A. S. Hardy · R. Roberts · M. Niedermeier · M. Kopp

Structural validation of three German versions of behavioral and motivational scales in high-risk sports

\section{Abstract}

The aim of the present research was to validate German language versions of three inventories in high-risk sports to facilitate future research in the significant population of German speaking highrisk sports participants. We translated the Sensation Seeking, Emotion Regulation and Agency Scale (SEAS), the Risk-Taking Inventory and the Accidents and Close Calls in Sport Inventory into German, then tested the hypothesized factor structures with 719 highrisk sport participants from the European Alps using Bayesian structural equation modelling (BSEM). The final models were all good fits to the data, had good internal consistency and displayed adequate discriminant validity. All inventories displayed the same factor structure as in the English inventories bar the G-SEAS After inventory in which a three- factor model fitted better than a two-factor model. Possible reasons for this difference include differences in the sample population, translation bias, or cross-cultural differences; however it seems likely that the nuanced approach of BSEM allowed this study to disentangle emotion regulation transfer from agency transfer after participating in high-risk sport where previous attempts using other methods have failed to. This will allow future research in high-risk sport to be conducted beyond English speaking populations and more significantly, facilitate the investigation of differences between the transfer effects of agency and emotion regulation.

\section{Keywords}

Risk-taking · Sensation seeking · Emotion regulation · Agency · Bayesian statistics

\section{Strukturelle Validierung dreier deutscher Versionen von Verhaltens- und Motivationsskalen für Hochrisikosportarten}

\section{Zusammenfassung}

Das Ziel der vorliegenden Untersuchung war es drei Versionen von Erhebungsinstrumenten im Hochrisikosport für die Verwendung bei deutschsprechenden Populationen zu validieren. Die Sensation Seeking, Emotion Regulation and Agency Scale (SEAS), das Risk-Taking Inventory und das Accidents and Close Calls in Sport Inventory wurden in die deutsche Sprache übersetzt. Die vermutete Faktorenstruktur wurde mit Strukturgleichungsmodellen der Bayesschen Statistik (BSEM) an 719 Hochrisikosportlern aus den europäischen Alpen getestet. Die finalen Modelle zeigten gute Werte für die Anpassungsgüte und für die interne Konsistenz sowie adäquate Werte für die Diskriminanzvalidität.Bis auf die G-SEAS Nach der Sportausübung wiesen alle Skalen die gleiche Faktorenstruktur wie die englischen Skalen auf. Im Gegensatz zu dem englischen Zwei-Faktoren-Modell in der SEAS Nach der Sportausübung zeigte sich ein Drei-FaktorenModell in der deutschen Skala. Mögliche
Gründe für diese Diskrepanz könnten Unterschiede in der Stichprobe, Übersetzungsbias oder interkulturelle Unterschiede sein. Allerdings erscheint es wahrscheinlich, dass der differenzierte Zugang der BSEM Berechnung dieser Studie erlaubte, den Transfer von Emotionsregulierung und wahrgenommener Kontrolle über die eigenen Handlungen (Agency) in der G-SEAS Nach der Sportausübung zu lösen. Angesichts der Validierungskennwerte können die Skalen für künftige Forschung im Hochrisikosport auch im deutschen Sprachraum empfohlen werden. Die drei-faktorielle Struktur der G-SEAS Nach der Sportausübung ermöglicht es zudem, etwaige Unterschiede zwischen den Transfereffekten von Agency und Emotionsregulierung herauszufiltern.

Schlüsselwörter

Risikoverhalten · Sensation Seeking · Emotionsregulierung $\cdot$ Agency $\cdot$ Bayessche Statistik 
Table 2 German Sensation Seeking, Emotion Regulation and Agency Scale (G-SEAS) Between items and standardized factor loadings with $95 \%$ credibility intervals (CI) in brackets

\begin{tabular}{|c|c|c|c|c|}
\hline $\begin{array}{l}\text { Original item (Barlow } \\
\text { et al., 2013) }\end{array}$ & German translation & $\begin{array}{l}\text { Sensation } \\
\text { seeking }\end{array}$ & $\begin{array}{l}\text { Emotion } \\
\text { regula- } \\
\text { tion }\end{array}$ & Agency \\
\hline $\begin{array}{l}\text { 1. I want to get an } \\
\text { adrenaline rush }\end{array}$ & $\begin{array}{l}\text { Ich möchte einen Adrenalinkick } \\
\text { bekommen }\end{array}$ & $\begin{array}{l}0.86 \\
{[0.73} \\
0.99]\end{array}$ & $\begin{array}{l}0.04 \\
{[-0.11} \\
0.19]\end{array}$ & $\begin{array}{l}-0.02 \\
{[-0.17} \\
0.13]\end{array}$ \\
\hline $\begin{array}{l}\text { 2. I look forward to get- } \\
\text { ting a physical thrill from } \\
\text { participating }\end{array}$ & $\begin{array}{l}\text { Ich erhoffe mir einen körperlich } \\
\text { wahrnehmbaren Nervenkitzel } \\
\text { beim Paragliding }\end{array}$ & $\begin{array}{l}0.90 \\
{[0.77} \\
1.02]\end{array}$ & $\begin{array}{l}-0.03 \\
{[-0.19} \\
0.12]\end{array}$ & $\begin{array}{l}0.01 \\
{[-0.15} \\
0.14]\end{array}$ \\
\hline $\begin{array}{l}\text { 3. I look forward to the } \\
\text { "rush" I hope to experience } \\
\text { while participating }\end{array}$ & $\begin{array}{l}\text { Ich freue mich auf den Kick, den } \\
\text { ich während der Ausübung zu } \\
\text { erleben hoffe }\end{array}$ & $\begin{array}{l}0.85 \\
{[0.69} \\
1.00]\end{array}$ & $\begin{array}{l}-0.03 \\
{[-0.19} \\
0.12]\end{array}$ & $\begin{array}{l}-0.01 \\
{[-0.17} \\
0.15]\end{array}$ \\
\hline $\begin{array}{l}6 . \text { I feel the need to do } \\
\text { something intensely stimu- } \\
\text { lating }\end{array}$ & $\begin{array}{l}\text { Ich habe das Verlangen, etwas } \\
\text { besonders Aufregendes zu tun }\end{array}$ & $\begin{array}{l}0.76 \\
{[0.57} \\
0.92]\end{array}$ & $\begin{array}{l}0.02 \\
{[-0.15} \\
0.19]\end{array}$ & $\begin{array}{l}0.03 \\
{[-0.13} \\
0.18]\end{array}$ \\
\hline $\begin{array}{l}\text { 7. The emotional elements } \\
\text { of my life are difficult to } \\
\text { deal with }\end{array}$ & $\begin{array}{l}\text { Es ist schwer mit emotionalen } \\
\text { Anteilen von meinem Leben } \\
\text { umzugehen }\end{array}$ & $\begin{array}{l}0.02 \\
{[-0.11} \\
0.15]\end{array}$ & $\begin{array}{l}0.85 \\
{[0.67} \\
1.04]\end{array}$ & $\begin{array}{l}0.03 \\
{[-0.15} \\
0.18]\end{array}$ \\
\hline $\begin{array}{l}\text { 8. I am emotional (e.g., } \\
\text { anxious, angry) without } \\
\text { understanding why }\end{array}$ & $\begin{array}{l}\text { Ich werde emotional (z.B. } \\
\text { ängstlich, wütend), ohne zu } \\
\text { wissen warum }\end{array}$ & $\begin{array}{l}-0.03 \\
{[-0.16} \\
0.10]\end{array}$ & $\begin{array}{l}0.92 \\
{[0.75} \\
1.10]\end{array}$ & $\begin{array}{l}-0.03 \\
{[-0.20} \\
0.13]\end{array}$ \\
\hline $\begin{array}{l}\text { 9. I struggle to deal with } \\
\text { stressful situations in my } \\
\text { life }\end{array}$ & $\begin{array}{l}\text { Ich habe Schwierigkeiten mit } \\
\text { Stresssituationen in meinem } \\
\text { Leben umzugehen }\end{array}$ & $\begin{array}{l}-0.02 \\
{[-0.14} \\
0.10]\end{array}$ & $\begin{array}{l}0.86 \\
{[0.69} \\
1.04]\end{array}$ & $\begin{array}{l}0.06 \\
{[-0.12} \\
0.21]\end{array}$ \\
\hline $\begin{array}{l}\text { 10. I can't work out which } \\
\text { emotion I am experiencing }\end{array}$ & $\begin{array}{l}\text { Ich kann nicht eindeutig zuord- } \\
\text { nen, welche Emotion ich ger- } \\
\text { ade erlebe }\end{array}$ & $\begin{array}{l}0.01 \\
{[0.14} \\
0.15]\end{array}$ & $\begin{array}{l}0.78 \\
{[0.57} \\
0.99]\end{array}$ & $\begin{array}{l}0.02 \\
{[-0.17} \\
0.19]\end{array}$ \\
\hline $\begin{array}{l}11 . \text { I find that emotional } \\
\text { situations in my life stress } \\
\text { me out }\end{array}$ & $\begin{array}{l}\text { Mich belasten emotionale } \\
\text { Situationen in meinem Leben }\end{array}$ & $\begin{array}{l}0.01 \\
{[-0.12} \\
0.01]\end{array}$ & $\begin{array}{l}0.89 \\
{[0.71} \\
1.08]\end{array}$ & $\begin{array}{l}-0.03 \\
{[-0.21} \\
0.14]\end{array}$ \\
\hline $\begin{array}{l}\text { 13. I am prevented from } \\
\text { achieving my goals in life }\end{array}$ & $\begin{array}{l}\text { Ich werde am Erreichen meiner } \\
\text { Lebensziele gehindert }\end{array}$ & $\begin{array}{l}0.04 \\
{[-0.10} \\
0.17]\end{array}$ & $\begin{array}{l}0.02 \\
{[-0.17} \\
0.21]\end{array}$ & $\begin{array}{l}0.78 \\
{[0.59} \\
0.98]\end{array}$ \\
\hline $\begin{array}{l}\text { 14. I feel like a passive } \\
\text { observer of my life rather } \\
\text { than a major "actor" }\end{array}$ & $\begin{array}{l}\text { Ich fühle mich eher als passiver } \\
\text { Beobachter meines Lebens und } \\
\text { weniger als aktiv steuernde } \\
\text { Person }\end{array}$ & $\begin{array}{l}-0.01 \\
{[-0.14} \\
0.11]\end{array}$ & $\begin{array}{l}0.02 \\
{[-0.16} \\
0.19]\end{array}$ & $\begin{array}{l}0.86 \\
{[0.68} \\
1.03]\end{array}$ \\
\hline $\begin{array}{l}\text { 16. I feel like my life "be- } \\
\text { longs" to other people }\end{array}$ & $\begin{array}{l}\text { Ich fühle mich, als ob andere } \\
\text { über mein Leben bestimmen }\end{array}$ & $\begin{array}{l}-0.01 \\
{[-0.13} \\
0.12]\end{array}$ & $\begin{array}{l}-0.06 \\
{[-0.23} \\
0.10]\end{array}$ & $\begin{array}{l}0.92 \\
{[0.76} \\
1.09]\end{array}$ \\
\hline 17. I feel trapped & Ich fühle mich gefangen & $\begin{array}{l}-0.07 \\
{[-0.21} \\
0.07]\end{array}$ & $\begin{array}{l}-0.03 \\
{[-0.21} \\
0.15]\end{array}$ & $\begin{array}{l}0.87 \\
{[0.67} \\
1.06]\end{array}$ \\
\hline $\begin{array}{l}\text { 18. I have little belief in my } \\
\text { own ability to influence } \\
\text { some important aspects of } \\
\text { my life }\end{array}$ & $\begin{array}{l}\text { Ich glaube nicht daran, dass ich } \\
\text { die Fähigkeit habe, wichtige } \\
\text { Aspekte meines Lebens zu } \\
\text { beeinflussen }\end{array}$ & $\begin{array}{l}0.05 \\
{[-0.08} \\
0.18]\end{array}$ & $\begin{array}{l}0.08 \\
{[-0.10} \\
0.25]\end{array}$ & $\begin{array}{l}0.77 \\
{[0.61} \\
0.96]\end{array}$ \\
\hline
\end{tabular}

ranging from one (never) to five (always). Internal consistency was evidenced by composite reliabilities of $0.64-0.78$ for DRT and $0.64-0.71$ for PB across three studies (Woodman et al., 2013). item, two-factor inventory asking about

\section{Accidents and Close Calls in Sport Inventory (ACCSI)}

The ACCSI (Barlow et al., 2015) is a sixexperienced accidents (three items) and close calls (three items) on a seven-point Likert scale from one (never) to seven (always). A good model fit was confirmed in varying samples (Barlow et al., 2015). Moderate correlations between DRT and Accidents $(\mathrm{r}=0.31-0.54)$ and Close Calls $(\mathrm{r}=0.52-0.64)$ were shown. PB showed weaker, negative correlations with Accidents $(\mathrm{r}=-0.02$ to -0.33$)$ and Close Calls $(r=-0.10$ to -0.34$)$.

\section{German scale development}

We translated the items following the guidelines of Guillemin, Bombardier, and Beaton (1993). The items were translated into German by a group of Sports Science Masters students, who were all fluent in German, and they were asked to note any remarks and questions while translating. The group met to discuss items and phrases until full consent was reached. In the next step translated and original items were sent to three experts from the field of health psychology and sport science who were equally fluent in both English and German language. They were asked if the German items were an accurate translation of the original English items, when they identified problematic items we modified them and the process was repeated until the experts agreed that all of the German items accurately represented the meaning of the original English ones.

\section{Statistical analyses}

We tested the hypothesized factor structure using Bayesian structural equation modelling (BSEM) in Mplus 7 (Muthén \& Muthén, 2012), estimating BSEMs with weakly informative priors for approximate zero cross-loadings and residual correlations, as recommended by Muthén and Asparouhov (2012), for each inventory. Each BSEM was estimated using the Markov chain Monte Carlo (MCMC) simulation procedure, using the Gibbs sampler over 200,000 iterations across two MCMC chains in order to assess model convergence and stability of estimates.

Model convergence can be assessed in a number of ways (Kaplan \& Depaoli, 2012). In this study, we used the GelmanRubin convergence diagnostic (potential scale reduction factor; PSR) and Kol- 
Table 3 German Sensation Seeking, Emotion Regulation and Agency Scale (G-SEAS) While items and standardized factor loadings with 95\% credibility intervals (CI) in brackets

\begin{tabular}{|c|c|c|c|c|}
\hline $\begin{array}{l}\text { Original item (Barlow } \\
\text { et al., 2013) }\end{array}$ & German translation & $\begin{array}{l}\text { Sensation } \\
\text { seeking }\end{array}$ & $\begin{array}{l}\text { Emotion } \\
\text { regula- } \\
\text { tion }\end{array}$ & Agency \\
\hline $\begin{array}{l}\text { 1. I enjoy getting a physi- } \\
\text { cal thrill }\end{array}$ & $\begin{array}{l}\text { Mir gefällt es, einen körperlich } \\
\text { wahrnehmbaren Nervenkitzel zu } \\
\text { bekommen }\end{array}$ & $\begin{array}{l}0.79 \\
{[0.56} \\
0.96]\end{array}$ & $\begin{array}{l}-0.06 \\
{[-0.22} \\
0.10]\end{array}$ & $\begin{array}{l}0.04 \\
{[-0.12} \\
0.20]\end{array}$ \\
\hline $\begin{array}{l}\text { 2. I experience intense } \\
\text { excitement }\end{array}$ & Ich bin sehr aufgeregt & $\begin{array}{l}0.72 \\
{[0.42} \\
0.94]\end{array}$ & $\begin{array}{l}0.03 \\
{[-015} \\
0.21]\end{array}$ & $\begin{array}{l}-0.06 \\
{[-0.23} \\
0.11]\end{array}$ \\
\hline $\begin{array}{l}5 . \text { I get a rush of chemical } \\
\text { around my body that } \\
\text { feels great }\end{array}$ & $\begin{array}{l}\text { Ich bekomme durch die Aus- } \\
\text { schüttung von körpereigenen } \\
\text { Substanzen einen Kick, der sich } \\
\text { großartig anfühlt }\end{array}$ & $\begin{array}{l}0.82 \\
{[0.62} \\
0.99]\end{array}$ & $\begin{array}{l}0.01 \\
{[-0.16} \\
0.16]\end{array}$ & $\begin{array}{l}-0.02 \\
{[-0.17} \\
0.13]\end{array}$ \\
\hline $\begin{array}{l}\text { 6. I experience physical } \\
\text { sensations that feel great }\end{array}$ & $\begin{array}{l}\text { lch erlebe körperliche Empfin- } \\
\text { dungen, die sich großartig an- } \\
\text { fühlen }\end{array}$ & $\begin{array}{l}0.65 \\
{[0.38} \\
0.91]\end{array}$ & $\begin{array}{l}0.06 \\
{[-0.12} \\
0.23]\end{array}$ & $\begin{array}{l}0.07 \\
{[-0.10} \\
0.23]\end{array}$ \\
\hline $\begin{array}{l}\text { 8. I have to deal with } \\
\text { stressful situations }\end{array}$ & $\begin{array}{l}\text { Ich muss mit Stresssituationen } \\
\text { umgehen können }\end{array}$ & $\begin{array}{l}-0.11 \\
{[-0.28} \\
0.08]\end{array}$ & $\begin{array}{l}0.71 \\
{[0.40} \\
0.94]\end{array}$ & $\begin{array}{l}0.03 \\
{[-0.16} \\
0.20]\end{array}$ \\
\hline $\begin{array}{l}10 . \text { I prove to myself that } \\
\text { I can deal with stressful } \\
\text { situations }\end{array}$ & $\begin{array}{l}\text { Ich beweise mir selbst, dass ich } \\
\text { mit Stresssituationen umgehen } \\
\text { kann }\end{array}$ & $\begin{array}{l}0.05 \\
{[-0.13} \\
0.21]\end{array}$ & $\begin{array}{l}0.77 \\
{[0.57} \\
0.97]\end{array}$ & $\begin{array}{l}0.04 \\
{[-0.13} \\
0.19]\end{array}$ \\
\hline $\begin{array}{l}\text { 11. I have to deal with } \\
\text { intense emotions }\end{array}$ & $\begin{array}{l}\text { Ich muss ich mit intensiven } \\
\text { Gefühlen umgehen }\end{array}$ & $\begin{array}{l}0.03 \\
{[-0.14} \\
0.18]\end{array}$ & $\begin{array}{l}0.84 \\
{[0.66} \\
1.01]\end{array}$ & $\begin{array}{l}-0.03 \\
{[-0.18} \\
0.12]\end{array}$ \\
\hline $\begin{array}{l}\text { 12. The emotions I expe- } \\
\text { rience are more intense } \\
\text { that in other areas of my } \\
\text { life }\end{array}$ & $\begin{array}{l}\text { Die Gefühle die ich erlebe sind in- } \\
\text { tensiver als in anderen Bereichen } \\
\text { meines Lebens }\end{array}$ & $\begin{array}{l}0.02 \\
{[-0.16} \\
0.19]\end{array}$ & $\begin{array}{l}0.73 \\
{[0.49} \\
0.96]\end{array}$ & $\begin{array}{l}0.00 \\
{[-0.17} \\
0.17]\end{array}$ \\
\hline $\begin{array}{l}\text { 13. If a difficult situation } \\
\text { arises I feel able to deal } \\
\text { with it }\end{array}$ & $\begin{array}{l}\text { Ich fühle ich mich in der Lage eine } \\
\text { schwierige Situation zu meistern, } \\
\text { wenn diese auftritt }\end{array}$ & $\begin{array}{l}0.04 \\
{[-0.12} \\
0.21]\end{array}$ & $\begin{array}{l}0.06 \\
{[-0.12} \\
0.23]\end{array}$ & $\begin{array}{l}0.59 \\
{[0.32} \\
0.82]\end{array}$ \\
\hline $\begin{array}{l}\text { 14. I am free from the } \\
\text { constraints imposed on } \\
\text { me in the rest of my life }\end{array}$ & $\begin{array}{l}\text { Ich bin frei von Einschränkungen } \\
\text { meines täglichen Lebens }\end{array}$ & $\begin{array}{l}0.01 \\
{[-0.16} \\
0.18]\end{array}$ & $\begin{array}{l}0.04 \\
{[-0.15} \\
0.22]\end{array}$ & $\begin{array}{l}0.61 \\
{[0.36} \\
0.86]\end{array}$ \\
\hline $\begin{array}{l}\text { 15. I choose how far to } \\
\text { push when I am scared }\end{array}$ & $\begin{array}{l}\text { Ich entscheide, wie weit ich in } \\
\text { Angstsituationen gehe }\end{array}$ & $\begin{array}{l}-0.00 \\
{[-0.16} \\
0.17]\end{array}$ & $\begin{array}{l}0.02 \\
{[-0.16} \\
0.18]\end{array}$ & $\begin{array}{l}0.71 \\
{[0.48} \\
0.92]\end{array}$ \\
\hline 16. I am in charge & Ich habe das Sagen & $\begin{array}{l}0.01 \\
{[-0.16} \\
0.17]\end{array}$ & $\begin{array}{l}-0.04 \\
{[-0.21} \\
0.13]\end{array}$ & $\begin{array}{l}0.74 \\
{[0.50} \\
0.92]\end{array}$ \\
\hline $\begin{array}{l}\text { 17. My actions and deci- } \\
\text { sions prevent undesired } \\
\text { outcomes from happen- } \\
\text { ing }\end{array}$ & $\begin{array}{l}\text { Meine Handlungen und Entschei- } \\
\text { dungen verhindern das Eintreten } \\
\text { unerwünschter Ereignisse }\end{array}$ & $\begin{array}{l}-0.05 \\
{[-0.21} \\
0.12]\end{array}$ & $\begin{array}{l}-0.02 \\
{[-0.20} \\
0.15]\end{array}$ & $\begin{array}{l}0.72 \\
{[0.50} \\
0.91]\end{array}$ \\
\hline $\begin{array}{l}\text { 18. No one can force me } \\
\text { to do something I don't } \\
\text { want to do }\end{array}$ & $\begin{array}{l}\text { Niemand kann mich zwingen } \\
\text { etwas zu tun, was ich nicht will }\end{array}$ & $\begin{array}{l}0.00 \\
{[-0.17} \\
0.17]\end{array}$ & $\begin{array}{l}-0.00 \\
{[-0.17} \\
0.17]\end{array}$ & $\begin{array}{l}0.65 \\
{[0.37} \\
0.86]\end{array}$ \\
\hline
\end{tabular}

mogorov-Smirnov (K-S) tests to assess model convergence in addition to visual inspection of all parameter trace plots. Model convergence is evident when the PSR value approaches one following the warm-up period (i.e., the first half of the iterations) (Gelman et al., 2014), when
K-S tests show no significant differences between the estimates of parameter distribution across the chains, and when parameter values in each MCMC chain mix well (i.e., have stable means and variances (van de Schoot \& Depaoli Sarah, 2014)).
We assessed model fit by examining: factor loadings, the posterior predictive $p(\mathrm{PP} p)$ value and the results of likelihood $\chi^{2}$ tests, which examine differences between the model generated and the observed data. Excellent model fit is indicated by $\mathrm{PP} p$ values of approximately 0.50 and with a symmetric 95\% credibility intervals (CI) centered around zero (Muthén \& Asparouhov, 2012). We assessed internal consistency using the composite reliability coefficient (Fornell \& Larcker, 1981) and discriminant validity using the latent variable correlations obtained from the BSEMs.

Where good model fit or convergence was not reached we re-examined the items in each scale to identify which items were problematic. To improve model fits, we removed items from each inventory; items were considered for removal based on the following criteria: having low factor loadings $(<0.6)$; theoretical (ir)relevance; having substantive cross-loadings or correlated residual variances $(> \pm 0.2)$; parameters with significant K-S tests; the highest PSR; or trace plots which showed poor mixing of the MCMC chains. Once identified, we removed these items and re-estimated the BSEM. We repeated this process until the final model for each inventory was both statistically and theoretically sound; it was important that item removal could be justified both statistically and theoretically to avoid making modifications based on sample specific, chance characteristics of the data, which then may not represent the relationships among variables in the wider population (Biddle, Markland, Gilbourne, Chatzisarantis, \& Sparkes, 2001).

Given that Barlow et al. (2013) found a two-factor structure to be most appropriate for the SEAS After inventory, we analyzed our data with two- and threefactor models in order to see if the translated version confirmed these initial findings. In addition to the criteria mentioned above, we used the Deviance Information Criteria (DIC) to compare the two- and three-factor G-SEAS After inventory as recommended by Asparouhov, Muthén, and Morin (2015).

Once the final models were established we performed a sensitivity analysis as the 
Table 4 German Sensation Seeking, Emotion Regulation and Agency Scale (G-SEAS) 3-factor model After items and standardized factor loadings with 95\% credibility intervals (CI) in brackets

\begin{tabular}{|c|c|c|c|c|}
\hline $\begin{array}{l}\text { Original item (Barlow } \\
\text { et al., 2013) }\end{array}$ & German translation & $\begin{array}{l}\text { Sensation } \\
\text { seeking }\end{array}$ & $\begin{array}{l}\text { Emotion } \\
\text { regula- } \\
\text { tion }\end{array}$ & Agency \\
\hline $\begin{array}{l}\text { 1. I enjoy the feeling } \\
\text { of adrenaline flowing } \\
\text { around my body }\end{array}$ & $\begin{array}{l}\text { Ich genieße es, wenn Adrenalin } \\
\text { durch meinen Körper strömt }\end{array}$ & $\begin{array}{l}0.90 \\
{[0.77} \\
1.05]\end{array}$ & $\begin{array}{l}-0.03 \\
{[-0.19} \\
0.13]\end{array}$ & $\begin{array}{l}-0.01 \\
{[-0.17} \\
0.13]\end{array}$ \\
\hline $\begin{array}{l}\text { 2. I feel like I have satis- } \\
\text { fied my immediate need } \\
\text { for thrill }\end{array}$ & $\begin{array}{l}\text { Ich habe das Gefühl, dass ich } \\
\text { mein Bedürfnis nach einem } \\
\text { Nervenkitzel gestillt habe }\end{array}$ & $\begin{array}{l}0.88 \\
{[0.72} \\
1.03]\end{array}$ & $\begin{array}{l}-0.02 \\
{[-0.18} \\
0.14]\end{array}$ & $\begin{array}{l}-0.02 \\
{[-0.18} \\
0.13]\end{array}$ \\
\hline $\begin{array}{l}\text { 3. I am often buzzing } \\
\text { from the adrenaline }\end{array}$ & $\begin{array}{l}\text { Ich bin oft vom Adrenalin be- } \\
\text { rauscht }\end{array}$ & $\begin{array}{l}0.83 \\
{[0.71} \\
1.00]\end{array}$ & $\begin{array}{l}0.01 \\
{[-0.15} \\
0.17]\end{array}$ & $\begin{array}{l}0.00 \\
{[-0.16} \\
0.15]\end{array}$ \\
\hline $\begin{array}{l}5 . \text { I enjoy the rush of } \\
\text { chemicals still flowing } \\
\text { round my body }\end{array}$ & $\begin{array}{l}\text { Ich genieße den Kick der aus- } \\
\text { geschütteten Substanzen, die } \\
\text { immer noch meinen Körper } \\
\text { durchfluten }\end{array}$ & $\begin{array}{l}0.87 \\
{[0.75} \\
1.01]\end{array}$ & $\begin{array}{l}0.02 \\
{[-0.13} \\
0.17]\end{array}$ & $\begin{array}{l}0.01 \\
{[-0.14} \\
0.15]\end{array}$ \\
\hline $\begin{array}{l}\text { 6. I look back and think } \\
\text { how much I enjoyed the } \\
\text { rush }\end{array}$ & $\begin{array}{l}\text { Ich blicke zurück und erinnere } \\
\text { mich daran, wie sehr ich den } \\
\text { Kick genossen habe }\end{array}$ & $\begin{array}{l}0.81 \\
{[0.65} \\
0.96]\end{array}$ & $\begin{array}{l}0.02 \\
{[-0.15} \\
0.18]\end{array}$ & $\begin{array}{l}0.03 \\
{[-0.13} \\
0.18]\end{array}$ \\
\hline $\begin{array}{l}\text { 9. I find intense emotions } \\
\text { easier to deal with }\end{array}$ & $\begin{array}{l}\text { Ich kann mit intensiven } \\
\text { Gefühlen besser umgehen }\end{array}$ & $\begin{array}{l}0.02 \\
{[-0.12} \\
0.15]\end{array}$ & $\begin{array}{l}0.88 \\
{[0.70} \\
1.08]\end{array}$ & $\begin{array}{l}0.02 \\
{[-0.16} \\
0.18]\end{array}$ \\
\hline $\begin{array}{l}10 . \text { I find it easier to deal } \\
\text { with stressful situations } \\
\text { in my life }\end{array}$ & $\begin{array}{l}\text { Ich kann mit Stresssituationen } \\
\text { im Alltag besser umgehen }\end{array}$ & $\begin{array}{l}-0.05 \\
{[-0.19} \\
0.09]\end{array}$ & $\begin{array}{l}0.90 \\
{[0.71} \\
1.11]\end{array}$ & $\begin{array}{l}0.02 \\
{[-0.17} \\
0.20]\end{array}$ \\
\hline $\begin{array}{l}\text { 11. I feel better for having } \\
\text { experienced my emo- } \\
\text { tions }\end{array}$ & $\begin{array}{l}\text { Ich fühle mich besser, nach- } \\
\text { dem ich meine Emotionen in } \\
\text { Erfahrung gebracht habe }\end{array}$ & $\begin{array}{l}0.09 \\
{[-0.06} \\
0.22]\end{array}$ & $\begin{array}{l}0.86 \\
{[0.66} \\
1.08]\end{array}$ & $\begin{array}{l}-0.04 \\
{[-0.22} \\
0.14]\end{array}$ \\
\hline $\begin{array}{l}\text { 12. I feel better able to } \\
\text { deal with aspects of my } \\
\text { life that would normally } \\
\text { make me feel emotional }\end{array}$ & $\begin{array}{l}\text { Ich kann besser mit Situatio- } \\
\text { nen umgehen, welche mich } \\
\text { üblicherweise emotional } \\
\text { berühren würden }\end{array}$ & $\begin{array}{l}-0.03 \\
{[-0.17} \\
0.10]\end{array}$ & $\begin{array}{l}0.89 \\
{[0.70} \\
1.09]\end{array}$ & $\begin{array}{l}0.04 \\
{[-0.15} \\
0.20]\end{array}$ \\
\hline $\begin{array}{l}\text { 13. I have a calmness that } \\
\text { carries over into other } \\
\text { aspects of my life }\end{array}$ & $\begin{array}{l}\text { Ich habe eine Gelassenheit in } \\
\text { mir, die sich auch auf andere } \\
\text { Lebensbereiche überträgt }\end{array}$ & $\begin{array}{l}-0.04 \\
{[-0.19} \\
0.12]\end{array}$ & $\begin{array}{l}-0.02 \\
{[-0.21} \\
0.18]\end{array}$ & $\begin{array}{l}0.75 \\
{[0.52} \\
0.98]\end{array}$ \\
\hline $\begin{array}{l}\text { 15. I am more confident } \\
\text { about facing challenges } \\
\text { in other aspects of my life }\end{array}$ & $\begin{array}{l}\text { Ich sehe anderen Heraus- } \\
\text { forderungen in meinem Leben } \\
\text { zuversichtlicher entgegen }\end{array}$ & $\begin{array}{l}-0.02 \\
{[-0.15} \\
0.10]\end{array}$ & $\begin{array}{l}0.02 \\
{[-0.16} \\
0.18]\end{array}$ & $\begin{array}{l}0.90 \\
{[0.74} \\
1.08]\end{array}$ \\
\hline $\begin{array}{l}\text { 16. I feel more influential } \\
\text { in how events in my life } \\
\text { unfold }\end{array}$ & $\begin{array}{l}\text { Ich habe das Gefühl, mehr Ein- } \\
\text { fluss auf die Entwicklung von } \\
\text { Ereignissen in meinem Leben zu } \\
\text { haben }\end{array}$ & $\begin{array}{l}0.04 \\
{[-0.08} \\
0.16]\end{array}$ & $\begin{array}{l}0.00 \\
{[-0.17} \\
0.16]\end{array}$ & $\begin{array}{l}0.89 \\
{[0.73} \\
1.07]\end{array}$ \\
\hline $\begin{array}{l}\text { 17. I feel better about } \\
\text { my ability to bring about } \\
\text { important outcomes in } \\
\text { my life }\end{array}$ & $\begin{array}{l}\text { Ich fühle mich gestärkt in } \\
\text { meiner Fähigkeit, bedeutsame } \\
\text { Ergebnisse in meinem Leben zu } \\
\text { erreichen }\end{array}$ & $\begin{array}{l}0.04 \\
{[-0.09} \\
0.16]\end{array}$ & $\begin{array}{l}-0.03 \\
{[-0.20} \\
0.13]\end{array}$ & $\begin{array}{l}0.93 \\
{[0.76} \\
1.09]\end{array}$ \\
\hline $\begin{array}{l}\text { 18. I feel more able to } \\
\text { prevent difficulties occur- } \\
\text { ring in other aspects of } \\
\text { my life }\end{array}$ & $\begin{array}{l}\text { Ich fühle mich besser im Stande } \\
\text { Schwierigkeiten in anderen } \\
\text { Lebensbereichen zu verhindern }\end{array}$ & $\begin{array}{l}-0.02 \\
{[-0.14} \\
0.10]\end{array}$ & $\begin{array}{l}0.06 \\
{[-0.12} \\
0.21]\end{array}$ & $\begin{array}{l}0.88 \\
{[0.72} \\
1.06]\end{array}$ \\
\hline
\end{tabular}

choice of priors can affect the parameter estimates (Muthén \& Asparouhov, 2012; Stenling, Ivarsson, Johnson, \& Lindwall, 2015). To do so, we re-ran the final models for each inventory with smaller (0.05) and larger (0.015) priors for the cross- loadings, before comparing the parameter estimates for discrepancies between these models and those estimates with a prior variance of 0.01 . We also weighted items with their respective factor loadings and then calculated Pearson's correlation coefficients to examine the relationships between subscale means.

\section{Results}

\section{Model fit and convergence}

Visual inspection of trace plots for all parameters supported convergence (i.e., showed good mixing and no upward or downward trends). In addition to this the PSR value for all models fell below 1.1 during the warm-up phase of the simulations and remained below 1.1 for the remaining iterations (•Table 1 ), and there were no significant K-S tests for any of the five models.

For each G-SEAS inventory, the initial 18-item BSEM models with non-informative priors achieved adequate convergence and all items had significant loadings on their intended factors only. However, there were a myriad of other problems that meant the models were not deemed to be suitable. In the Between inventory, two items had problematic correlated residuals and one low major factor loading (0.49). In the While inventory, there were ten problematic correlated residuals and three major factor loadings were low (0.53-0.58). The three-factor After inventory had five problematic correlated residuals and one low major factor loading (0.56). The two-factor After model had one low major factor loading (0.56) and had 17 correlated residuals that exceeded their a priori limits. Through an iterative process, four items were removed from each G-SEAS inventory using the criteria outlined earlier (the remaining items can be seen in 0 Tables 2,3 and 4).

The BSEMs for the three 14-item G-SEAS inventories, the G-ACCSI, and the G-RTI with informative small variance priors for cross-loadings and residual correlations have excellent fit with $\mathrm{PP} p$ values of approximately 0.5 and having symmetric 95\% posterior predictive CIs centered on zero (• Table 1$)$. The major factor loadings in each inventory were significant, acceptable, and in the hypothesized direction. Furthermore, there were no cross-loadings that exceeded their a priori limits (- Tables 2, 3, 4, 5 and 6). There were 
Table 5 German Risk-Taking Inventory (G-RTI) items and standardized factor loadings with 95\% credibility intervals (CI) in brackets

\begin{tabular}{|c|c|c|c|}
\hline $\begin{array}{l}\text { Original item (Woodman } \\
\text { et al., 2013) }\end{array}$ & German translation & $\begin{array}{l}\text { Deliberate } \\
\text { Risk-Taking }\end{array}$ & $\begin{array}{l}\text { Precautionary } \\
\text { Behaviors }\end{array}$ \\
\hline $\begin{array}{l}\text { 1. I deliberately put myself in } \\
\text { danger }\end{array}$ & $\begin{array}{l}\text { Ich bringe mich bewusst } \\
\text { in Gefahr }\end{array}$ & $0.81[0.48,0.97]$ & $\begin{array}{l}0.01[-0.17, \\
0.18]\end{array}$ \\
\hline $\begin{array}{l}\text { 2. It's like gambling, you can't } \\
\text { win unless you try it }\end{array}$ & $\begin{array}{l}\text { Es ist wie im Glücksspiel: } \\
\text { wer nicht probiert, kann } \\
\text { auch nicht gewinnen }\end{array}$ & $0.66[0.24,0.92]$ & $\begin{array}{l}-0.02[-0.20 \\
0.17]\end{array}$ \\
\hline $\begin{array}{l}\text { 3. I actively seek out danger- } \\
\text { ous situations }\end{array}$ & $\begin{array}{l}\text { Ich suche gezielt } \\
\text { gefährliche Situationen } \\
\text { auf }\end{array}$ & $0.81[0.54,0.95]$ & $\begin{array}{l}0.00[-0.17, \\
0.17]\end{array}$ \\
\hline $\begin{array}{l}\text { 4. I take time to check condi- } \\
\text { tions (e.g., weather) }\end{array}$ & $\begin{array}{l}\text { Ich nehme mir Zeit, die } \\
\text { Umweltbedingungen zu } \\
\text { überprüfen (z. B.: Wetter) }\end{array}$ & $\begin{array}{l}-0.01[-0.18, \\
0.18]\end{array}$ & $0.72[0.36,0.91]$ \\
\hline $\begin{array}{l}5 . \text { I check any gear/equipment } \\
\text { that I borrow }\end{array}$ & $\begin{array}{l}\text { Ich überprüfe jeden } \\
\text { Ausrüstungsgegenstand, } \\
\text { den ich mir ausleihe }\end{array}$ & $\begin{array}{l}0.01[-0.16, \\
0.18]\end{array}$ & $0.75[0.47,0.93]$ \\
\hline $\begin{array}{l}\text { 6. I am aware of the nearest } \\
\text { help and first aid }\end{array}$ & $\begin{array}{l}\text { Ich weiß, wo sich die } \\
\text { nächste Servicestelle und } \\
\text { erste Hilfe befindet }\end{array}$ & $\begin{array}{l}0.03[-0.15 \\
0.21]\end{array}$ & $0.69[0.26,0.90]$ \\
\hline $\begin{array}{l}\text { 7. I take time to check for } \\
\text { potential hazards }\end{array}$ & $\begin{array}{l}\text { Ich nehme mir die Zeit, } \\
\text { mich über mögliche } \\
\text { Gefahren zu informieren }\end{array}$ & $\begin{array}{l}-0.04[-0.21 \\
0.13]\end{array}$ & $0.77[0.53,0.93]$ \\
\hline
\end{tabular}

Table 6 German Accidents and Close Calls in Sports Inventory (G-ACCSI) items and standardized factor loadings with $95 \%$ credibility intervals (CI) in brackets

\begin{tabular}{|c|c|c|c|c|}
\hline Factor & $\begin{array}{l}\text { Original item (Barlow } \\
\text { et al., 2015) }\end{array}$ & German translation & Accidents & $\begin{array}{l}\text { Close } \\
\text { Calls }\end{array}$ \\
\hline \multirow[t]{3}{*}{$\begin{array}{l}\text { Close } \\
\text { Calls }\end{array}$} & $\begin{array}{l}\text { 1. I experience close calls } \\
\text { when participating in my } \\
\text { sport }\end{array}$ & $\begin{array}{l}\text { Ich entgehe Unfällen bei der } \\
\text { Ausübung meines Sports nur } \\
\text { knapp }\end{array}$ & $\begin{array}{l}0.82 \\
{[0.54} \\
1.01]\end{array}$ & $\begin{array}{l}-0.01 \\
{[-0.20,} \\
0.17]\end{array}$ \\
\hline & $\begin{array}{l}\text { 2. I find myself in situa- } \\
\text { tions that lead to near } \\
\text { misses }\end{array}$ & $\begin{array}{l}\text { Ich erlebe Situationen, die } \\
\text { beinahe in Unfällen münden }\end{array}$ & $\begin{array}{l}0.80 \\
{[0.55} \\
0.99]\end{array}$ & $\begin{array}{l}0.02 \\
{[-0.17} \\
0.21]\end{array}$ \\
\hline & $\begin{array}{l}\text { 3. During participation } \\
\text { in my sport, I narrowly } \\
\text { avoid accidents }\end{array}$ & $\begin{array}{l}\text { Bei der Ausübung meines Sports } \\
\text { gehe ich bis ans Limit wo Unfälle } \\
\text { gerade noch vermieden werden } \\
\text { können }\end{array}$ & $\begin{array}{l}0.77 \\
{[0.48} \\
0.99]\end{array}$ & $\begin{array}{l}0.01 \\
{[-0.19} \\
0.20]\end{array}$ \\
\hline \multirow[t]{3}{*}{ Accidents } & $\begin{array}{l}\text { 4. I am involved in acci- } \\
\text { dents when participating } \\
\text { in my sport }\end{array}$ & $\begin{array}{l}\text { Bei der Ausübung meines Sports } \\
\text { bin ich in Unfälle involviert }\end{array}$ & $\begin{array}{l}0.04 \\
{[-0.16} \\
0.23]\end{array}$ & $\begin{array}{l}0.84 \\
{[0.62} \\
1.02]\end{array}$ \\
\hline & $\begin{array}{l}\text { 5. My decisions in this } \\
\text { activity lead to accidents }\end{array}$ & $\begin{array}{l}\text { Meine Entscheidungen in dieser } \\
\text { Aktivität führen zu Unfällen }\end{array}$ & $\begin{array}{l}-0.02 \\
{[-0.21} \\
0.16]\end{array}$ & $\begin{array}{l}0.84 \\
{[0.60} \\
1.03]\end{array}$ \\
\hline & $\begin{array}{l}6 . \text { I sustain injuries as } \\
\text { a result of accidents }\end{array}$ & $\begin{array}{l}\text { Unfälle führen dazu, dass ich mir } \\
\text { Verletzungen zuziehe }\end{array}$ & $\begin{array}{l}-0.02 \\
{[-0.21} \\
0.18]\end{array}$ & $\begin{array}{l}0.76 \\
{[0.45} \\
0.97]\end{array}$ \\
\hline
\end{tabular}

no correlated residuals that exceeded their a priori bounds in the Between, While, three-factor After participation G-SEAS, G-RTI, or G-ACCSI. However, there were four correlated residuals that exceeded their a priori limits in the twofactor After inventory all in the Agentic
The DIC for the 14-item two-factor After model was 19,097.742 and the DIC for the 14-item, three-factor After model was $19,096.132$, lower than that of the two-factor model despite having 16 more parameters. Despite the differences in DIC being small, the three-factor After model is a better fit to the data as there are no correlated residuals that exceed their a priori limits.

\section{Model sensitivity}

Sensitivity analyses for each inventory showed that the factor loadings and cross-loadings were relatively stable when specifying smaller (0.005) and larger (0.015) a priori variance priors. Using both smaller and larger prior variances $100 \%$ of discrepancies across the three inventories were within \pm 0.05 , the maximum discrepancy was -0.043 with smaller prior variances set; and the maximum discrepancy was -0.029 with larger prior variances set.

\section{Internal consistency, discriminant validity, and concurrent validity}

- Table 7 shows the latent factor subscale means, standard deviations, composite reliabilities and latent factor intercorrelations for the three G-SEAS inventories, the G-RTI, and the G-ACCSI. The composite reliability of each subscale resulted in $r>0.8$ across each inventory and the subscales within each inventory were all positively correlated. The G-RTI subscales had a weak inverse relationship and the Accidents and Close Call subscales were positively correlated (0.61). None of the $95 \%$ CIs for interfactor correlations encompassed 1.00, thus, supporting the discriminant validity of the subscales within each inventory (Anderson \& Gerbing, 1988).

- Table 8 shows correlations between subscale means, where the items were weighted with factor loadings. Sensation seeking of the Between participation G-SEAS was positively correlated with DRT, accidents and close calls and negatively with PB and age. Accidents and close calls were positively correlated with sensation seeking, which was also negatively related to both $\mathrm{PB}$ and age. 
Table 7 Latent factor subscale means, SDs, composite reliabilities and latent factor intercorrelations $(95 \% \mathrm{Cl})$

\begin{tabular}{|c|c|c|c|c|c|c|}
\hline Scale & Subscale & $\mathbf{M}$ & SD & CR & 1 & 2 \\
\hline \multirow{3}{*}{$\begin{array}{l}\text { G-SEAS } \\
\text { Between }\end{array}$} & 1 Sensation seeking & 3.00 & 1.46 & 0.91 & - & - \\
\hline & 2 Emotion regulation & 2.39 & 1.48 & 0.94 & $\begin{array}{l}0.49[0.32 \\
0.64]^{* * *}\end{array}$ & - \\
\hline & 3 Agency & 2.30 & 1.44 & 0.93 & $\begin{array}{l}0.34[0.21 \\
0.56]^{* * *}\end{array}$ & $\begin{array}{l}0.79[0.68, \\
0.86]^{* * *}\end{array}$ \\
\hline \multirow[t]{3}{*}{ G-SEAS While } & 1 Sensation seeking & 3.20 & 1.11 & 0.84 & - & - \\
\hline & 2 Emotion regulation & 3.52 & 1.15 & 0.85 & $\begin{array}{l}0.48[0.27 \\
0.66]^{* * *}\end{array}$ & - \\
\hline & 3 Agency & 3.62 & 0.75 & 0.83 & $\begin{array}{l}0.38[0.16 \\
0.57]^{* * *}\end{array}$ & $\begin{array}{l}0.51[0.31, \\
0.67]^{* * *}\end{array}$ \\
\hline \multirow{3}{*}{$\begin{array}{l}\text { G-SEAS After } \\
\text { 3-factor }\end{array}$} & 1 Sensation seeking & 3.44 & 1.50 & 0.94 & - & - \\
\hline & 2 Emotion regulation & 3.65 & 1.55 & 0.95 & $\begin{array}{l}0.59[0.44 \\
0.72]^{* * *}\end{array}$ & - \\
\hline & 3 Agency & 3.88 & 1.44 & 0.94 & $\begin{array}{l}0.53[0.36 \\
0.67]^{* *}\end{array}$ & $\begin{array}{l}0.83[0.74, \\
0.89]^{* * *}\end{array}$ \\
\hline \multirow[t]{2}{*}{ G-RTI } & $\begin{array}{l}1 \text { Deliberate risk- } \\
\text { taking }\end{array}$ & 4.21 & 1.94 & 0.80 & - & - \\
\hline & $\begin{array}{l}2 \text { Precautionary be- } \\
\text { haviors }\end{array}$ & 12.43 & 2.11 & 0.82 & $\begin{array}{l}-0.22[-0.43 \\
0.05]\end{array}$ & - \\
\hline \multirow[t]{2}{*}{ G-ACCSI } & 1 Accidents & 4.86 & 2.63 & 0.84 & - & - \\
\hline & 2 Close calls & 6.10 & 2.69 & 0.85 & $\begin{array}{l}0.61[0.41 \\
0.77]^{* * * *}\end{array}$ & - \\
\hline \multicolumn{7}{|c|}{$\begin{array}{l}\text { M Mean, SD Standard Deviation, CR Composite Reliabilities, } 95 \% \text { CI 95\% Credibility Interval, } \\
\text { G-SEAS German Sensation Seeking, Emotion Regulation and Agency Scale, G-ACCSI German } \\
\text { Accidents and Close Calls in Sports Inventory, G-RTI German Risk-Taking Inventory } \\
{ }^{* *} p<0.01,{ }^{* * *} p<0.001\end{array}$} \\
\hline
\end{tabular}

Accidents and close calls were both positively correlated with DRT and negatively with $\mathrm{PB}$. Age displayed negative correlations with all subscales except $\mathrm{PB}$, which resulted in a weak positive relation.

Difficulty with emotion regulation as measured by the Between participation emotion regulation subscale, correlated positively with DRT, accidents, and close calls and negatively with $\mathrm{PB}$ and age.

\section{Discussion}

The aim of this study was to validate three different German versions of inventories in high-risk sports. The BSEM analyses supported a good model fit in all three scales (G-SEAS, G-RTI, G-ACCSI). All subscales of each inventory showed good internal consistency and supported good discriminant validity. The correlations between the G-RTI and G-ACCSI scales derived similar results as shown in the original paper (Barlow et al., 2015). While in the development of the original RTI and the ACCSI the rela- tionship with the SEAS inventories was not tested, Barlow et al. (2015) tested the relationship between the original RTI, ACCSI, and alexithymia (i.e., difficulty describing, feeling, or identifying emotions). The relationships between alexithymia and the RTI and ACCSI were similar to the relationships between the Between emotion regulation subscale and the G-RTI and G-ACCSI subscales. These correlations suggest that a difficulty with emotion regulation is associated with higher deliberate risktaking (i.e., the positive correlation of the G-SEAS Between emotion regulation and DRT and its negative correlation with PB (•Table 8)). Deliberate risktaking in the G-RTI correlated with the experience of Sensation Seeking in the G-SEAS as much as DRT in the RTI correlated with the Brief Sensation Seeking Scale (Hoyle, Stephenson, Palmgreen, Lorch, \& Donohew, 2002) in the study by Barlow et al., (2015). This suggests that there is a link between the motivations for participation in high-risk sport and participants' behaviors.

The factor structure of the original scales was replicated in the G-RTI and G-ACCSI (Barlow et al., 2015; Woodman et al., 2013) with similar factor loadings in the G-ACCSI and higher factor loadings in the G-RTI. Similarly, the factor structure of the original SEAS was replicated in the Between and While inventory of the G-SEAS replicated the factor structure of the original SEAS; Sensation Seeking, Emotion Regulation and Agency were shown as separate constructs (Barlow et al., 2013). This supports the view of a multidimensional construct of motivation in high-risk sports. In the analysis of the After inventory of the G-SEAS a threefactor BSEM was a better fit to the data than a two-factor BSEM as was found in the original SEAS After inventory, with satisfaction of sensation needs and agentic emotion regulation transfer as the two factors.

Four possible reasons for the difference in factor structure between the SEAS and G-SEAS are as follows: differences in analytical methods, translation bias, cross-cultural differences, and differences in sports included in the samples. Contrary to the SEAS analyses, the present analyses used BSEM, which allows more complex, and thus more realistic, models to be specified. BSEM was recently used by Niven and Markland (2016) to establish motivational factors in walking and was favored over the independent clusters model using a maximum likelihood approach to confirmatory factor analysis (MLCFA ICM). The authors criticized the ML-CFA ICM approach because "ICM approach channels unspecified covariation between indicators through their factors, upwardly biasing interfactor correlations" (Niven \& Markland, 2016, p. 97). This artificial inflation of interfactor correlations may be the reason why Barlow et al. (2013) rejected the threefactor model in the original SEAS After inventory. Given that emotion regulation and agency are conceptually related, a highly restrictive model (e.g., ML-CFA ICM) would be less suitable from a theoretical standpoint than a model that does allow for small cross-loadings and 
Table 8 Weighted Pearson's correlations between the Between participation G-SEAS subscales, G-RTI subscales, G-ACCSI subscales, and age

\begin{tabular}{|c|c|c|c|c|c|c|c|}
\hline & 1 & 2 & 3 & 4 & 5 & 6 & 7 \\
\hline 1 Sensation Seeking & - & - & - & - & - & - & - \\
\hline 2 Emotion Regulation & $0.469^{* * *}$ & - & - & - & - & - & - \\
\hline 3 Agency & $0.374^{* * *}$ & $0.766^{* * *}$ & - & - & - & - & - \\
\hline 4 Deliberate Risk Taking & $0.476^{* * *}$ & $0.286^{* * *}$ & $0.217^{* * *}$ & - & - & - & - \\
\hline 5 Precautionary Behaviors & $-0.139^{* * *}$ & $-0.128^{* * *}$ & $-0.136^{* *}$ & $-0.205^{* * *}$ & - & - & - \\
\hline 6 Close Calls & $0.495^{* * *}$ & $0.324^{* * *}$ & $0.228^{* * *}$ & $0.462^{* * *}$ & $-0.135^{* *}$ & - & - \\
\hline 7 Accidents & $0.331^{* * *}$ & $0.227^{* * *}$ & $0.140^{* * *}$ & $0.353^{* * *}$ & $-0.109^{* *}$ & $0.577^{* * *}$ & - \\
\hline 8 Age & $-0.394^{* * *}$ & $-0.279^{* * *}$ & $-0.223^{* * *}$ & $-0.278^{* * *}$ & $0.141^{* * *}$ & $-0.238^{* * *}$ & $-0.138^{* * *}$ \\
\hline \multicolumn{8}{|c|}{$\begin{array}{l}\text { G-SEAS German Sensation Seeking, Emotion Regulation and Agency Scale, G-ACCSI German Accidents and Close Calls in Sports Inventory, G-RTI German } \\
\text { Risk-Taking Inventory } \\
{ }^{* *} p<0.01,{ }^{* * *} p<0.001 \text { (2-tailed) }\end{array}$} \\
\hline
\end{tabular}

residual correlations (e.g., BSEM with weakly informative priors). We only analyzed the data using BSEM; therefore, we cannot conclude how, if at all, factor structures may have been different when using ML-CFA ICM. However, if these differences in the factor structure are due differences in the analytical methods it may be that BSEM could reveal a threefactor structure in the After inventory of the original SEAS data.

Another possible explanation for the differences in items and factor structure could be due to translation bias and/or cross-cultural differences. Validation studies of translated health constructs repeatedly showed differences in factor structure and number of items (Hwang, Kim, Kim, Kim, \& Ahn, 2013; Kim, DeCoster, Huang, \& Bryant, 2013; Nagels et al., 2013). Cross-cultural differences were especially noticed between different ethnic populations, e.g., Korean and Greek (Hwang et al., 2013) or Hispanics and non-Hispanics (Sanchez \& Vargas, 2016).

It is now evident that high-risk sport participants are not one homogenous population (Barlow et al., 2015, 2013; Castanier et al., 2010; Woodman et al., 2010); therefore, it is possible that differences in sports included the sample populations may account for the differences in factor structures between the G-SEAS and SEAS. The sample used by Barlow et al. (2013) included skydivers, mountaineers and low-risk sport participants; the sample in this study included paragliders, mountain runners, freeride skiers, freestyle skiers, and mountaineers.
It was shown in the health surveillance of clinical populations that analyzing different clinical samples resulted in a different factor structure (Thaler et al., 2015). In the present study, participants from a wide range of high-risk sports have been included, going some way towards reflecting the heterogeneity of high-risk sport populations, thus, making the questionnaire usable for a large population.

The differences of the G-SEAS to the SEAS should not be considered as a negative outcome since the constructs showed a good model fit and the adjustments of the G-SEAS (shorter questionnaire, three factors for the After inventory) could be interpreted as improvements to the scale. Shorter questionnaires have higher response rates (Nakash, Hutton, JørstadStein, Gates, \& Lamb, 2006); therefore, response rates and adherence to the 14item scale may be higher than to an 18item scale. From a measurement perspective the three-factor structure of the After inventory presented in this article identifies emotion regulation and agency as two distinct transfer mechanisms in the current sample. Barlow et al. (2013) showed, using the two-factor solution in the After inventory that mountaineers experience a significant higher agentic emotion regulation transfer than both skydivers and low-risk controls. While participating, only the experience of emotion regulation could distinguish between mountaineers and skydivers which means that skydivers experienced similar feelings of agency while participating than mountaineers. This raises the question whether skydivers experience an agency transfer effect, which could not be shown in the study by Barlow et al. (2013) due to confounding results of emotion regulation and the limitation of a two-factor structure. Duration of the activity may be more important to the transfer of emotion regulation than agency. Freeride skiing comprises elements of thrill seeking (using lift-supported access for the activity) as well as a prolonged activity (using ski touring/hiking for the activity). Frühauf et al. (2017) reported that freeriders appreciate the fact that they are in charge of what they do and that they are not forced to follow strict rules (i.e., experience agency) and for some, this was crucial to their wellbeing (e.g., transfer effect of agency). The three-factor model in the G-SEAS After inventory might contribute to a better understanding of transfer effects from high-risk sports by offering the possibility to distinguish between agency and emotion regulation transfer effects in the German-speaking population for future studies using the G-SEAS.

\section{Strengths and limitations}

First, one might mention a lack of validation with established measures (e.g., the Sensation Seeking Scale). However, the concurrent validity of the inventories has been well established in the original since we validated constructs which already put those inventories in relation to established measures (Barlow et al., 2013; Woodman et al., 2013), validating the three scales with one another was seen as sufficient. Second, both the 
exclusion of SEAS items and structural validation was done in the same sample. This approach might result in biased estimates for structural validation. Future studies using the G-SEAS might consider calculating indices for internal consistency and factorial structure. As discussed there are several strengths resulting from the differences in the factor structure of the G-SEAS; nevertheless, a limiting factor is that because of those differences, the G-SEAS and the original SEAS cannot be used to directly compare German- and English-speaking populations. This study has provided evidence for a three-factor structure for each of the G-SEAS scales. Without collecting new data in both German- and Englishspeaking populations to carry out a measurement invariance analysis, it is not possible to make direct comparisons between data collected using the SEAS and G-SEAS. We would recommend that this analysis be carried out using BSEM as the differences in factor structures of the SEAS and G-SEAS may be due to differences in analytic methodologies. Therefore, we recommend that G-SEAS should be used in German-speaking populations and should not be used to make direct comparisons with data collected using the SEAS. Interscale correlations do not differ between the 14-item and the 18item German version; this suggests that despite missing 4 items, the G-SEAS still measures identical constructs. Another limitation which is not necessarily tied to this research only is the in our opinion problematic operationalization of highrisk sports. Despite the definition we cited at the beginning of the introduction, we think future research might benefit from a more detailed definition including additional components related to skills and experience. Though this is beyond the scope of this article, it is worth mentioning that researchers in high-risk sports need to address this problem in future studies.

This study has a number of strengths, including the variation within the population; in addition to being of sufficient size, the sample included a number of different high-risk sports, participants from a variety of experience levels and of different ages and sexes, thus, ensuring a het- erogenic sample population. The analytical methods used in this study should also be seen as another strength of this article. It has been shown that BSEM is a more appropriate method than traditional CFA methods as it better reflects the complexities of reality (Niven \& Markland, 2016; Stenling et al., 2015).

\section{Conclusion}

The current study validated three different inventories for high-risk sports in German language (G-SEAS, G-RTI, G-ACCSI) and showed good internal consistency, discriminant validity, and a good model fit in all scales. The BSEM analyzes support a 14 -item three-factor structure of the G-SEAS despite the SEAS having a 18 -item three-factor structure for While and Between and a two-factor structure for the After participation inventory (Barlow et al., 2013). The G-SEAS is seen as an improvement to the SEAS since shorter questionnaires increase response rates (Nakash et al., 2006) and a three-factor structure might help to distinguish between agency and emotion regulation transfer effects in future studies. However, a limiting factor of the differences in the factor structure is that the G-SEAS and SEAS cannot be used to directly compare German- and English-speaking populations with one another. Correlation analyses displayed relations between motivational and behavioral components of the high-risk sport activity with Sensation Seeking showing the highest correlation with deliberate risk-taking. The present study validated the three different inventories in German language, which is a first step towards the development of crosscultural motivational and behavioral constructs in high-risk sport participants.

\section{Corresponding address}

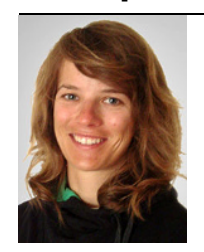

A. Frühauf

Department of Sport Science, University of Innsbruck Fürstenweg 185, 6020 Innsbruck, Austria anika.fruehauf@uibk.ac.at
Acknowledgements. The authors would like to thank Robin Owen and Dr. Sophie Wilmes for help with translation.

Funding. Open access funding provided by University of Innsbruck and Medical University of Innsbruck.

\section{Compliance with ethical guidelines}

Conflict of interest. A. Frühauf, W.A.S. Hardy, R. Roberts, M. Niedermeier and M. Kopp declare that they have no competing interests.

The study was approved by the institutional Board for Ethical Questions in accordance with the Declaration of Helsinki.

Open Access. This article is distributed under the terms of the Creative Commons Attribution 4.0 International License (http://creativecommons.org/licenses/by/ 4.0/), which permits unrestricted use, distribution, and reproduction in any medium, provided you give appropriate credit to the original author(s) and the source, provide a link to the Creative Commons license, and indicate if changes were made.

\section{References}

Anderson, J.C., \& Gerbing, D.W. (1988). Structural equation modeling in practice: a review and recommended two-step approach. Psychological Bulletin, 103(3), 411-423. https://doi.org/10. 1037/0033-2909.103.3.411.

Asparouhov, T., Muthén, B., \& Morin, A.J.S. (2015). Bayesian structural equation modeling with cross-loadings and residual covariances. Journal of Management, 41(6), 1561-1577. https://doi. org/10.1177/0149206315591075.

Barlow, M., Woodman, T., \& Hardy, L. (2013). Great expectations: different high-risk activities satisfy different motives. Journal of personality and social psychology, 105(3), 458-475. https://doi. org/10.1037/a0033542.

Barlow, M., Woodman, T., Chapman, C., Milton, M. Stone, D., Dodds, T., \& Allen, B. (2015). Who takes risks in high-risk sport? The role of alexithymia. Journal of sport \& exercise psychology, 37(1), 83-96. https://doi.org/10.1123/jsep.20140130.

Biddle, S. J., Markland, D., Gilbourne, D., Chatzisarantis, N. L., \& Sparkes, A.C. (2001). Research methods in sport and exercise psychology: quantitative and qualitative issues. Journal of sports sciences, 19(10), 777-809. https://doi.org/10.1080/ 026404101317015438.

Breivik, G. (1999). Empirical studies of risk sport. Oslo: Norges Idrettshøgskole. Institutt for samfunnsfag.

Brymer, E. (2010). Risk taking in extreme sports: a phenomenological perspective. Annals of Leisure Research, 13(1-2), 218-238. https://doi. org/10.1080/11745398.2010.9686845.

Brymer, E., \& Gray, T. (2010). Developing an intimate "relationship" with nature through extreme sports participation. Leisure/Loisir, 34(4), 361-374. https://doi.org/10.1080/14927713. 2010.542888

Burtscher, M. (2008). Risk of cardiovascular events during mountain activities. In R.C. Roach, P.D. 
Wagner \& P.H. Hackett (Eds.), Hypoxia and the circulation 1st edn. Advances in Experimental Medicine and Biology, (Vol. 618, pp. 1-11). Berlin, Heidelberg: Springer. https://doi.org/10. 1007/978-0-387-75434-5 1.

Castanier, C., Le Scanff, C., \& Woodman, T. (2010). Who takes risks in high-risk sports? A typological personality approach. Research quarterly for exercise and sport, 81(4), 478-484. https://doi. org/10.1080/02701367.2010.10599709.

Castanier, C., Le Scanff, C., \& Woodman, T. (2011). Mountaineering as affect regulation: the moderating role of self-regulation strategies. Anxiety, stress, and coping, 24(1), 75-89. https:// doi.org/10.1080/10615801003774210.

Fornell, C., \& Larcker, D. F. (1981). Evaluating structural equation models with unobservable variables and measurement error. Journal of Marketing Research, 18(1), 39. https://doi.org/10.2307/ 3151312.

Frühauf, A., Hardy, W.A.S., Pfoestl, D., Hoellen, F.G., \& Kopp, M. (2017). A qualitative approach on motives and aspects of risks in freeriding. Frontiers in Psychology, 8, 141. https://doi.org/ 10.3389/fpsyg.2017.01998.

Gelman, A., Vehtari, A., Dunson, D. B., Rubin, D. B., Stern, H. S., \& Carlin, J. B. (2014). Bayesian data analysis (3rd edn.). Texts in statistical science. Boca Raton: CRC Press. Retrieved from http://proquest.tech. safaribooksonline.de/9781439898222

Gosteli, G., Yersin, B., Mabire, C., Pasquier, M., Albrecht, R., \& Carron, P.-N. (2016). Retrospective analysis of 616 air-rescue trauma cases related to the practice of extreme sports. Injury, 47(7), 1414-1420. https://doi.org/10.1016/j.injury. 2016.03.025.

Guillemin, F., Bombardier, C., \&Beaton, D. (1993). Crosscultural adaptation of health-related quality of life measures: literature review and proposed guidelines. Journal of clinical epidemiology, 46(12), 1417-1432.

Hoyle, R.H., Stephenson, M.T., Palmgreen, P., Lorch, E.P., \& Donohew, R. (2002). Reliability and validity of a brief measure of sensation seeking. Personality and Individual Differences, 32(3), 401-414. https://doi.org/10.1016/S01918869(01)00032-0.

Hwang, S. S.-H., Kim, E. Y., Kim, S. H., Kim, Y.S., \& Ahn, Y.M. (2013). Intercultural differences in factor structure of the SWN-20 in patients with schizophrenia. Comprehensive psychiatry, 54(7), 970-973. https://doi.org/10.1016/j.comppsych. 2013.04.001.

Kaplan, D., \& Depaoli, S. (2012). Bayesian structural equation modelling. In R.H. Hoyle (Ed.), Handbook of structural equation modelling. New York: Guilford.

Kerr, J. H., \& Mackenzie, H. S. (2012). Multiple motives for participating in adventure sports. Psychology of Sport and Exercise, 13(5),649-657. https://doi. org/10.1016/j.psychsport.2012.04.002.

Kim, G., DeCoster, J., Huang, C.-H., \& Bryant, A.N. (2013). A meta-analysis of the factor structure of the Geriatric Depression Scale (GDS): the effects of language. International psychogeriatrics, 25(1), 71-81. https://doi.org/ 10.1017/S1041610212001421.

Lafollie, D., \& Le Scanff, C. (2007). Détection des personnalités à risque dans les sports à sensations fortes. L'Encéphale, 33(2), 135-141. https://doi. org/10.1016/S0013-7006(07)91543-2.

Leiter, A.M., \& Rheinberger, C.M. (2016). Risky sports and the value of safety information. Journal of Economic Behavior \& Organization,
131, 328-345. https://doi.org/10.1016/j.jebo. 2016.09.003.

Llewellyn, D. J., \& Sanchez, X. (2008). Individual differences and risk taking in rock climbing. Psychology of Sport and Exercise, 9(4), 413-426. https://doi.org/10.1016/j.psychsport.2007.07. 003.

Martínez-González, M. A., Varo, J. J., Santos, J.L., de Irala, J., Gibney, M., Kearney, J., \& Martínez, J. A (2001). Prevalence of physical activity during leisure time in the European Union. Medicine and science in sports and exercise, 33(7), 1142-1146.

Mata, R., Josef, A. K., \& Hertwig, R. (2016). Propensity for risk taking across the life span and around the globe. Psychological science, 27(2), 231-243. https://doi.org/10.1177/0956797615617811.

Molinaro, S., Canale, N., Vieno, A., Lenzi, M., Siciliano, V., Gori, M., \& Santinello, M. (2014). Countryand individual-level determinants of probable problematic gambling in adolescence: a multilevel cross-national comparison. Addiction (Abingdon, England), 109(12), 2089-2097. https://doi.org/10.1111/add.12719.

Muthén, B., \& Asparouhov, T. (2012). Bayesian structural equation modeling: a more flexible representation of substantive theory. Psychological methods, 17(3), 313-335. https://doi.org/10. 1037/a0026802.

Muthén, L. K., \& Muthén, B. O. (1998-2012). Mplus User's Guide (7th ed.). Los Angeles, CA: Muthén \& Muthén.

Nagels, A., Stratmann, M., Ghazi, S., Schales, C., Frauenheim, M., Turner, L., et al. (2013). The German translation and validation of the scale for the assessment of thought, language and communication: a factor analytic study. Psychopathology, 46(6), 390-395. https://doi. org/10.1159/000345359.

Nakash, R. A., Hutton, J. L., Jørstad-Stein, E. C., Gates, S., \& Lamb, S.E. (2006). Maximising response to postal questionnaires - a systematic review of randomised trials in health research. $B M C$ medical research methodology, 6, 5. https://doi. org/10.1186/1471-2288-6-5.

Niven, A.G., \& Markland, D. (2016). Using selfdetermination theory to understand motivation forwalking: instrument developmentand model testing using Bayesian structural equation modelling. Psychology of Sport and Exercise, 23, 90-100. https://doi.org/10.1016/j.psychsport. 2015.11.004.

Pain, M. T.G., \& Pain, M. A. (2005). Essay: risk taking in sport. The Lancet, 366, S33-S34. https://doi.org/ 10.1016/S0140-6736(05)67838-5.

Sanchez, G.R., \& Vargas, E.D. (2016). Language bias and self-rated health status among the Latino population: evidence of the influence of translation in a wording experiment. Quality of life research, 25(5), 1131-1136. https://doi.org/ 10.1007/s11136-015-1147-8.

Stenling, A., Ivarsson, A., Johnson, U., \& Lindwall, M. (2015). Bayesian structural equation modeling in sport and exercise psychology. Journal of sport \& exercise psychology, 37(4), 410-420. https://doi. org/10.1123/jsep.2014-0330.

Thaler, N.S., Barchard, K. A., Parke, E., Jones, W. P., Etcoff, L. M., \&Allen, D. N. (2015). Factor structure of the Wechsler intelligence scale for children: fourth edition in children with ADHD. Journal of attention disorders, 19(12), 1013-1021. https:// doi.org/10.1177/1087054712459952.

Turner, C., McClure, R., \& Pirozzo, S. (2004). Injury and risk-taking behavior-a systematic review. Accident Analysis \& Prevention,
36(1), 93-101. https://doi.org/10.1016/S00014575(02)00131-8.

Van de Schoot, R., \& Depaoli, S. (2014). Bayesian analyses: where to start and what to report. European Health Psychologist, 16, 75-84.

Wallén Warner, H., Ozkan, T., \& Lajunen, T. (2009). Cross-cultural differences in drivers' speed choice. Accident; analysis and prevention, 41(4), 816-819. https://doi.org/10.1016/j.aap.2009. 04.004 .

Willig, C. (2008). A phenomenological investigation of the experience of taking part in 'extreme sports. Journal of health psychology, 13(5), 690-702. https://doi.org/10.1177/1359105307082459.

Woodman, T., Huggins, M., Le Scanff, C., \& Cazenave, N. (2009). Alexithymia determines the anxiety experienced in skydiving. Journal of affective disorders, 116(1-2), 134-138. https://doi.org/10 1016/j.jad.2008.11.022.

Woodman, T., Hardy, L., Barlow, M., \& Le Scanff, C. (2010). Motives for participation in prolonged engagement high-risk sports: an agentic emotion regulation perspective. Psychology of Sport and Exercise, 11(5), 345-352. https://doi. org/10.1016/j.psychsport.2010.04.002.

Woodman, T., Barlow, M., Bandura, C., Hill, M., Kupciw, D., \& Macgregor, A. (2013). Not all risks are equal: the risk taking inventory for high-risk sports. Journal of sport \& exercise psychology 35(5), 479-492.

Zuckerman, M. (1994). Behavioral expressions and biosocial bases of sensation seeking. New York: Cambridge University Press.

Zuckerman, M. (2008). Sensation seeking and risky behavior (p. 2). Washington, DC: American Psychological Assoc.. 